\title{
Interrupting vaccination policies can greatly spread SARS-CoV-2 and enhance mortality from COVID-19 disease: the AstraZeneca case for France and Italy
}

Article

Accepted Version

Faranda, D., Alberti, T., Arutkin, M., Lembo, V. and Lucarini, V. ORCID: https://orcid.org/0000-0001-9392-1471 (2021) Interrupting vaccination policies can greatly spread SARSCoV-2 and enhance mortality from COVID-19 disease: the AstraZeneca case for France and Italy. Chaos: An Interdisciplinary Journal of Nonlinear Science, 31 (4). 041105. ISSN 1089-7682 doi: https://doi.org/10.1063/5.0050887 Available at https://centaur.reading.ac.uk/97622/

It is advisable to refer to the publisher's version if you intend to cite from the work. See Guidance on citing.

To link to this article DOI: http://dx.doi.org/10.1063/5.0050887

Publisher: American Institute of Physics

All outputs in CentAUR are protected by Intellectual Property Rights law, including copyright law. Copyright and IPR is retained by the creators or other copyright holders. Terms and conditions for use of this material are defined in the End User Agreement. 


\section{www.reading.ac.uk/centaur}

\section{CentAUR}

Central Archive at the University of Reading

Reading's research outputs online 


\title{
Interrupting vaccination policies can greatly spread SARS-CoV-2 and enhance mortality from COVID-19 disease: the AstraZeneca case for France and Italy
}

\author{
Davide Faranda* \\ Laboratoire des Sciences du Climat et de l'Environnement, \\ CEA Saclay l'Orme des Merisiers, UMR 8212 CEA-CNRS-UVSQ, \\ Université Paris-Saclay \& IPSL, 91191, Gif-sur-Yvette, France. \\ London Mathematical Laboratory, 8 Margravine Gardens, London, W6 8RH, UK. and \\ LMD/IPSL, Ecole Normale Superieure, \\ PSL research University, 75005, Paris, France. \\ Tommaso Alberti \\ National Institute for Astrophysics-Institute for \\ Space Astrophysics and Planetology (INAF-IAPS), \\ via del Fosso del Cavaliere 100, 00133, Rome, Italy. \\ Maxence Arutkin \\ UMR CNRS 7083 Gulliver, ESPCI Paris, \\ 10 rue Vauquelin, 75005 Paris, France. \\ Valerio Lembo \\ National Research Council of Italy, \\ Institute of Atmospheric Sciences and Climate (CNR-ISAC), Bologna, Italy. \\ Valerio Lucarini \\ Department of Mathematics and Statistics, \\ University of Reading, Reading, UK. and \\ Centre for the Mathematics of Planet Earth, \\ University of Reading, Reading, UK.
}

(Dated: March 30, 2021) 


\begin{abstract}
Several European countries have suspended the inoculation of the AstraZeneca vaccine out of suspicion of causing deep vein thrombosis. In this letter we report some Fermi estimates performed using a stochastic model aimed at making a risk-benefit analysis of the interruption of the delivery of the AstraZeneca vaccine in France and Italy. Our results clearly show that excess deaths due to the interruption of the vaccination campaign injections largely overrun those due to thrombosis even in worst case scenarios of frequency and gravity of the vaccine side effects.
\end{abstract}

\footnotetext{
*davide.faranda@cea.fr
} 
We analyze, in the framework of epidemiological modelling, the stop in the deployment of the AstraZeneca vaccine due to some suspected side effects. Indeed, few dozen suspicious cases of Deep Vein Thrombosis (DVT) over 5 millions vaccinations have arisen in Europe and pushed several European countries to suspend AstraZeneca injection. Using both an epidemiological SusceptibleExposed-Infected-Recovered (SEIR) model and statistical analysis of publicly available data, we estimate the excess deaths resulting from missing inoculations of the vaccine and those potentially linked to DVT side effects in France and Italy. We find that, despite the many simplifications and limitations in our analysis, the excess deaths differ by at least an order of magnitude in the two strategies, that the relative benefits are wider in situations where the reproduction number is larger, and they increase with the temporal duration of the vaccine ban.

\section{INTRODUCTION}

As of March 2021, the spread of the SARS-CoV-2 virus [1] has caused more than 120 millions infections worldwide with a total death toll of more than 2 millions. Up to the end of 2020, the only effective measures to contain the spread of the virus were based on social distancing, wearing face masks and more/less stringent lockdown [2-4]. Later on, a massive vaccination campaign kicked off in several countries thanks to the availability of a variety of vaccines (e.g., AstraZeneca, Johnson\&Johnson, Moderna, Pfizer/BionTech, Sputnik V). Such vaccines differ substantially in terms of efficacy, legal status, availability, and logistics needed for their delivery to patients. According to various estimates [5], vaccinations would produce a reduction in infections, and eventually yield to "herd immunity" when $\approx 70 \%$ of the population gets fully vaccinated. When such a large fraction of the population becomes immune to the disease, its spread from person to person becomes very unlikely, and the whole community becomes protected. By allowing for an earlier easing of non-medical measures against the SARS-CoV-2 virus, vaccination is also expected to significantly reduce the economical, social and psychological impacts of lockdown measures [6]. Those estimates 
assume that there is no break in the supply of vaccines or any other suspension in the procedure due to side effects from vaccination. Unfortunately, on March 15th 2021 several European countries suspended the use of AstraZeneca COVID-19 vaccine as a precaution in order to investigate the death of a few dozens of patients developing blood clots - associated with Deep Vein Thrombosis (DVT) [7] - after such vaccine, despite no proof has been found yet of causal link between vaccination and DVT [8]. Health personnel who inoculated the vaccine to those who died as a result of DVT are being investigated in Italy for manslaughter [9]. The contingent situation with the widespread COVID-19 pandemic naturally raises the question of whether a prolonged stop in vaccinations coming from adopting the precautionary principle [10] could cause an excess mortality beyond that caused by hypothetical side effects of the vaccines. The European Medicines Agency (EMA) is currently assessing whether the vaccine can continue to be used despite possibly causing this very rare side effect. In this Letter, we aim at exploring this issue by computing future COVID-19 epidemic scenarios by comparing i) the excess mortality caused by reducing the vaccinations using the stochastic Susceptible-Exposed-Infected-Recovered (SEIR) model [11], and ii) the estimates of the possible casualties caused by side effects of a vaccine, namely those associated with DVT. We remark that the additional, longer-term effect of the presence of higher infection rates, e.g. the increased risk of virus mutations leading to possibly more malignant and/or more infectious variants, is not included in our treatment. Our analysis focuses on France and Italy, which have been among the countries that have been most severely impacted by the COVID-19 pandemic [12]. An important remark follows. Our goal is not to provide an exact estimate of both i) and ii) but rather to perform an order-of-magnitude comparison between excess deaths resulting from different scenarios of vaccination policy. We proceed in the spirit of complexity science, where simple models are useful for elucidating the main mechanisms behind complex behaviour and provide useful inputs for the deployment of more advanced modelling suites and data collection strategies [13-17]. In other words, we will approach the problem by performing Fermi estimates [18] where the classical back-of-theenvelope calculations are performed via the SEIR model, allowing to take into account the uncertainties in both model parameters and data. In nuce, we perform a counterfactual analysis based on a story-line approach, which has become a powerful investigation method for assessing risks coming from extreme events [19]. While the quantitative consolidation of our results clearly requires extensive data analysis and modelling, our findings show with 
a large confidence that excess deaths due to the interruption of the vaccination campaign largely override those due to DVT even in the worst case scenarios of frequency and gravity of the vaccine side effects. Fermi estimates can provide valuable inputs for an efficient and pragmatic application of the precautionary principle able to reduce the negative impacts of hazards of various nature, as done in economics [20].

\section{METHODS}

The model [21] with time-dependent control parameters can mimic the dependence on additional/external factors such as variability in the detected cases, different physiological response to the virus, release or reinforcement of distancing measures [11]. Our compartmental model [22] divides the population into four groups, namely Susceptible (S), Exposed (E), Infected (I), and Recovered (R) individuals, according to the following evolution equations:

$$
\begin{aligned}
S_{t+1} & =S_{t}-\lambda(1-\alpha) \frac{I_{t} S_{t}}{N_{t}}-\lambda \alpha(1-\sigma) \frac{I_{t} S_{t}}{N_{t}} \\
& +(1-\sigma \alpha) S_{t} \\
E_{t+1} & =E_{t}+\lambda(1-\alpha) \frac{I_{t} S_{t}}{N_{t}}+\lambda \alpha(1-\sigma) \frac{I_{t} S_{t}}{N_{t}} \\
& +(1-\epsilon) E_{t} \\
I_{t+1} & =I_{t}+\epsilon E_{t}+(1-\alpha-\beta) I_{t} \\
R_{t+1} & =R_{t}+\sigma \alpha S_{t}+\beta I_{t}
\end{aligned}
$$

In the SEIR model above, the classical parameters are the recovery rate $(\beta)$, the inverse of the incubation period $(\epsilon)$, and the infection rate $(\lambda)$. Here we have generalized the model presented in Faranda and Alberti [11] by introducing two additional parameters able to succinctly mimic the strategies of a vaccination campaign, namely the vaccination rate per capita $\alpha$ and the vaccine efficacy $\sigma$, see Sun and Hsieh [23]. In order to consider uncertainties in long-term extrapolations and time-dependent control parameters, a stochastic approach is used through which the control parameters $\kappa \in\{\alpha, \beta, \epsilon, \lambda, \sigma\}$ are described by an OrnsteinUhlenbeck process [24] with drift as follows:

$$
\mathrm{d} \kappa=-\kappa(t) \mathrm{d} t+\kappa_{0} \mathrm{~d} t+\varsigma_{\kappa} d W_{t},
$$


where $\kappa_{0} \in\left\{\alpha_{0}, \beta_{0}, \epsilon_{0}, \lambda_{0}, \sigma_{0}\right\}, d W_{t}$ is the increment of a Wiener process. We remind that the basic reproduction number [25] is written as $R_{0}=\beta_{0} / \lambda_{0}$. In Eqs. (1)-(5) we set $d t=1$, which is the highest time resolution available for official COVID-19-related counts and is relatively small compared to the characteristic times associated with COVID-19 infection, incubation, and recovery/death.

\begin{tabular}{|c|c|c|c|c|}
\hline$\alpha_{0}$ & $\beta_{0}$ & $\epsilon_{0}$ & $\sigma_{0}$ & $m_{0}$ \\
0.0015 [see Ref. 26] & 0.37 [see Ref. 11] & 0.27 [see Ref. 27] & 0.59 [see Ref. 28] & 0.015 [see Ref. 29] \\
\hline$\varsigma_{\alpha}$ & $\varsigma_{\beta}$ & $\varsigma_{\epsilon}$ & $\varsigma_{\sigma}$ & $\varsigma_{m}$ \\
0.25 [see Ref. 26] & 0.2 [see Ref. 21] & 0.2 [see Ref. 26] & 0.1 [see Ref. 28] & 0.0 \\
\hline
\end{tabular}

TABLE I. Model parameters used for our simulations with corresponding references.

Initialising parameters with their associated reference are shown in Table I. The mortality rate $m_{0}$ is also shown, set to 0.015 [29]. While $\beta_{0}$ and $\epsilon_{0}$ and the associated $\varsigma$ are the same as in [11], the values of $\sigma_{0}$ and respective $\varsigma$ are derived from the range given for the AstraZeneca vaccine phase 3 tests for the first dose [28], and $\alpha_{0}$ and $\varsigma_{\alpha}$ are given supposing that both Italy and France keep vaccinating $10^{5}$ individuals per day with a $20 \%$ daily fluctuation [26]. As in [11], we also set $\varsigma_{\lambda}=0.2$, allowing for $20 \%$ daily fluctuations in the infection rate. Note that here we restrict to Gaussian fluctuations: as shown in [11], allowing for log-normal fluctuations of the parameters does not change the average results but slightly enhance their dispersion. See Supplementary Material for the numerical code.

\section{ESTIMATE OF THE EXCESS DEATHS DUE TO STOPPING ASTRAZENECA VACCINE INOCULATION}

Figure 1 reports the daily number of deaths $m_{0} \times I_{t}$ as a function of time for Italy (a) and France (b). Initial conditions are set for both countries to the values reported on March 15th as follows: for Italy, we set $N=60 \cdot 10^{6}$ population, $E_{t=1}=I_{t=1}=20 \cdot 10^{4}$ as the infected and exposed populations, $R_{t=1}=11 \cdot 10^{6}$ as the sum of $9 \cdot 10^{6}$ recovered estimated from serologic tests and $2 \cdot 10^{6}$ immunized from 2 doses of either Pfizer/BioNTech, Moderna or AstraZeneca vaccines and $R_{0}=1.16$. For France, we set $N=67 \cdot 10^{6}, E_{t=1}=I_{t=1}=25 \cdot 10^{4}$, $R_{t=1}=13.2 \cdot 10^{6}$ as the sum of $11 \cdot 10^{6}$ recovered estimated from serologic tests and $2.2 \cdot 10^{6}$ immunized from vaccines and $R_{0}=1.02$. For both France and Italy, we assume that the 
virus, after the second wave, has infected the $15 \%$ of the population. This estimates are based on Pullano et al. [30] who reported a 7\% $3 \%$ total infections for France after the first wave, assuming that the second wave had a similar magnitude for both countries. We remark however, that our results are basically insensitive to oscillation of $S(1)$ of order of 5 millions individuals (cfr. Supplementary Material Figure S1). Rather than integrating the Fokker-Planck equation [31] corresponding to the system of equations given above, we follow a Monte Carlo approach and we perform two sets of $N_{r}=1000$ realizations (see supplementary material Figure S2 for a justification of this value): stopping (red) and continuing (blue) the vaccination campaign at the same rate. The model is integrated for 500 days, that is about the time it would take to vaccine the rest of the susceptible population with AstraZeneca at the rate of $10^{5}$ individuals per day.

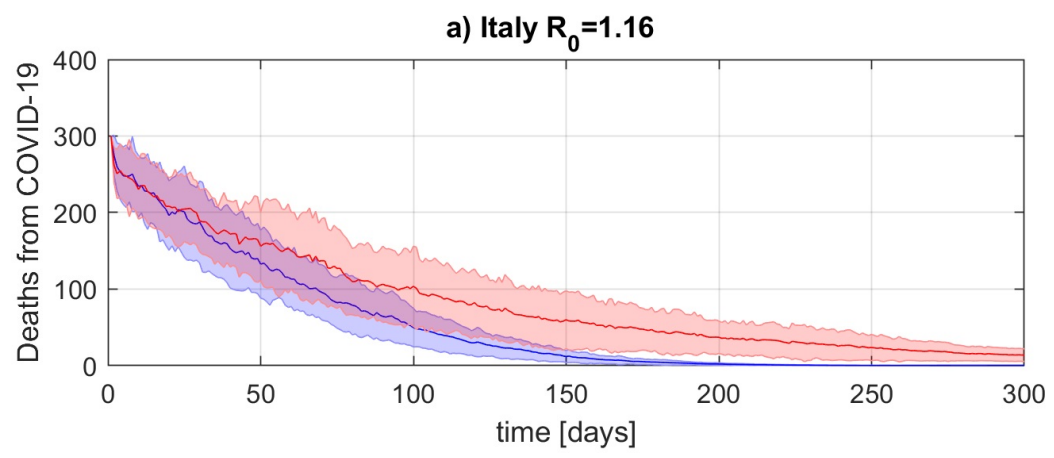

b) France $R_{0}=1.02$

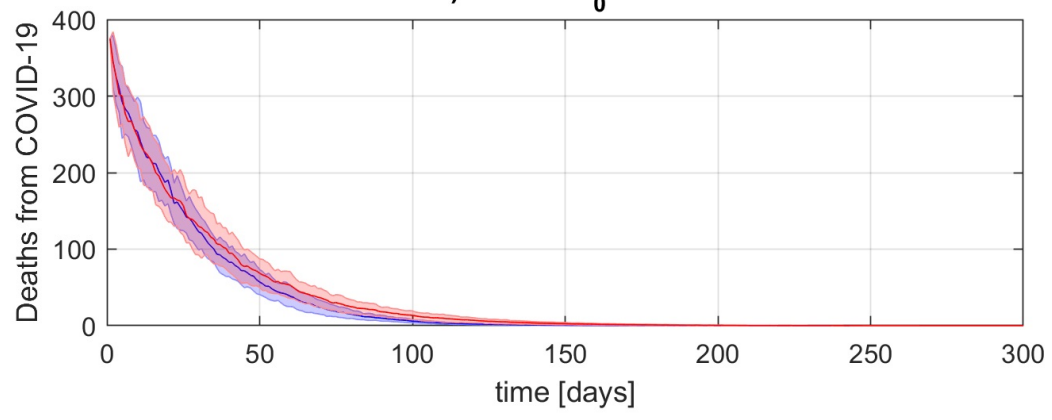

FIG. 1. The number of daily deaths $m \times I(t)$ as a function of time (300 out of 500 days shown) for Italy (a) and France (b) using the values of $R_{0}=1.16$ (Italy) and $R_{0}=1.02$ reported respectively for the 15th of March countries. Solid lines show the ensemble average, dotted lines extend to one standard deviation of the mean. Red and blue curves refer respectively to no vaccination and a vaccination campaign whose efficacy is $59 \%$.

First, we observe a monotonic decrease in the daily deaths for all scenarios considered 
from the initial date $t=t_{0}$ corresponding to March 15, 2021. This is in agreement with actual estimates that for Italy and France the so-called third wave should reach its peak in the second half of March, 2021 [32]Moreover, we observe that the cumulative number of deaths significantly (we take the width of the error bars as level of significance) reduces if vaccinations are continued at 100000 doses per day with respect to the scenario where vaccination is stopped. For Italy (France) completely halting the vaccination, at the actual epidemic rate, the number of excess deaths from COVID19 would amount to $9 \pm 3 \cdot 10^{3}$ $\left(1.2 \pm 0.4 \cdot 10^{3}\right)$ excess deaths from COVID19. The difference between the two countries is largely due to the value of $R_{0}$, which is larger for Italy. This suggests that halting vaccination in a growing epidemics phase (Italy) has more dramatic consequences than in a more controlled scenario of $R_{0} \approx 1$ (France).

Our previous analysis is based on a total stop of AstraZeneca vaccination. However, a more realistic scenario is to assume that AstraZeneca vaccination will resume after a limited number of days used for verification. We investigate this effect in Fig. 2. There, we consider the average excess deaths as a function of the interruption length in number of days (x-axis) and $R_{0}$ (y-axis) for Italy (a) and France (b). The excess deaths are computed with respect to a base scenario where vaccine injections are never interrupted and they are averaged over 1000 realizations of the SEIR model. Figure 2 shows that the longer is the vaccine injections disruption, the higher is the number of excess deaths. The impact is stronger for higher values of $R_{0}$. While waiting the advice of EMA about AstraZeneca safety, many national health agencies also announced that, when allowed, they would resume the vaccination at a higher rate than before to override the effects of the stop. In the supplementary Figure S3 we therefore present a set of simulation where, for a number of days equal to those of the vaccination interruption, injections are performed at a double rate than originally planned, i.e., $2 \cdot 10^{5}$ individuals/day, in order to compensate for the lost vaccinations. Although reduced, the number of excess deaths is still high and of the same order of magnitude as the one estimated in Fig. 2, as a result of the nonlinear cascade effect of the extra infections occurred in the period when vaccinations were interrupted. A focus on the actual values of $R_{0}$ for Italy and France is reported in Fig. 3. Here we compare the two countries and we also show the effect of doubling vaccination rates. This shows that excess deaths scale down by a factor two but they remain of the same order of magnitude as for the case of a business-as-usual vaccination rate, namely $10^{5}$ vaccinations/day. 

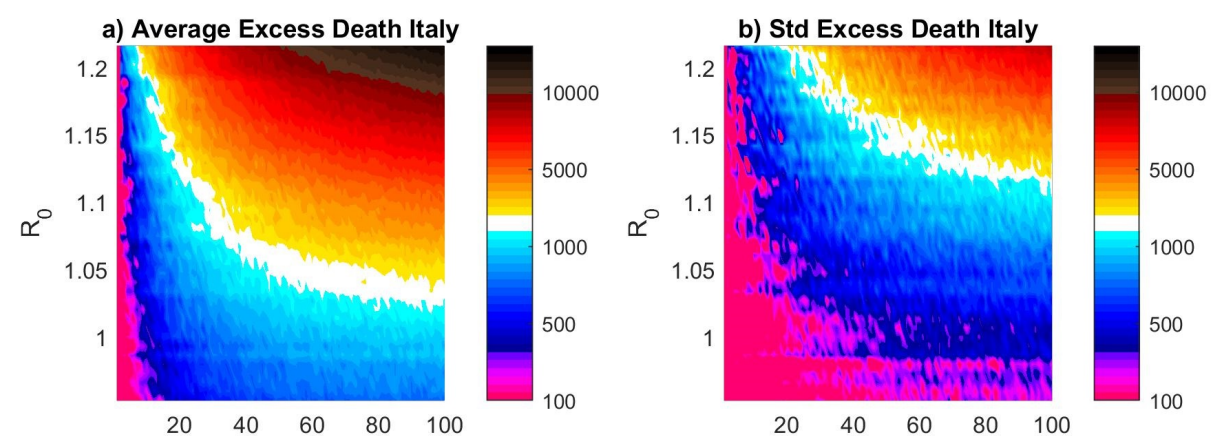

Number of consecutive days of vaccine interruption Number of consecutive days of vaccine interruption

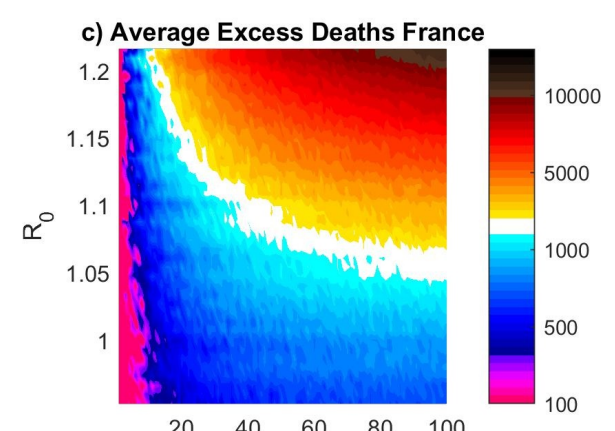

$20 \quad 40 \quad 60 \quad 80 \quad 100$

Number of consecutive days of vaccine interruption

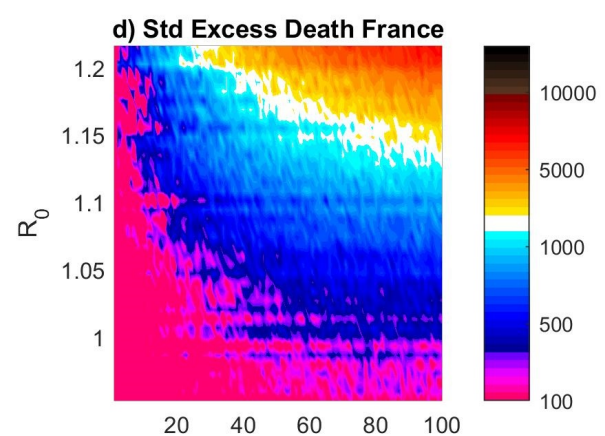

Number of consecutive days of vaccine interruption

FIG. 2. (a,c) Average and (b,d) standard deviation over $N_{r}=1000$ realizations of the stochastic SEIR model showing the excess deaths $m \times I(t)$ as a function of the number of the days of interruption of AstraZeneca vaccinations (x-axis) and $R_{0}$ (y-axis) for Italy $(\mathrm{a}, \mathrm{b})$ and France $(\mathrm{c}, \mathrm{d})$. The excess deaths are computed with respect to a base scenario where vaccine injections are never interrupted. Note that $x$-axis starts at $N=1$. Each realization of the SEIR model is integrated for 500 days.

\section{WORST CASE SCENARIOS FOR ASTRAZENECA SIDE EFFECTS}

The final step in our investigation is to compare the previous estimates of excess deaths with an order of magnitude estimate of deaths due to DVT resulting from side effects of the AstraZeneca vaccine. In order to make a meaningful comparison, in a case where uncertainties are very large and hard to quantify, we will consider a worst case scenario for the impacts of the side effects. This scenario relies on the unrealistic hypothesis that the totality of susceptible population to DVT suffers from DVT shortly after being vaccinated, and the lethality rate is similar to the one observed in the overall population.

As of March 15th 2021, few dozens suspect cases of DVT have been reported over a 


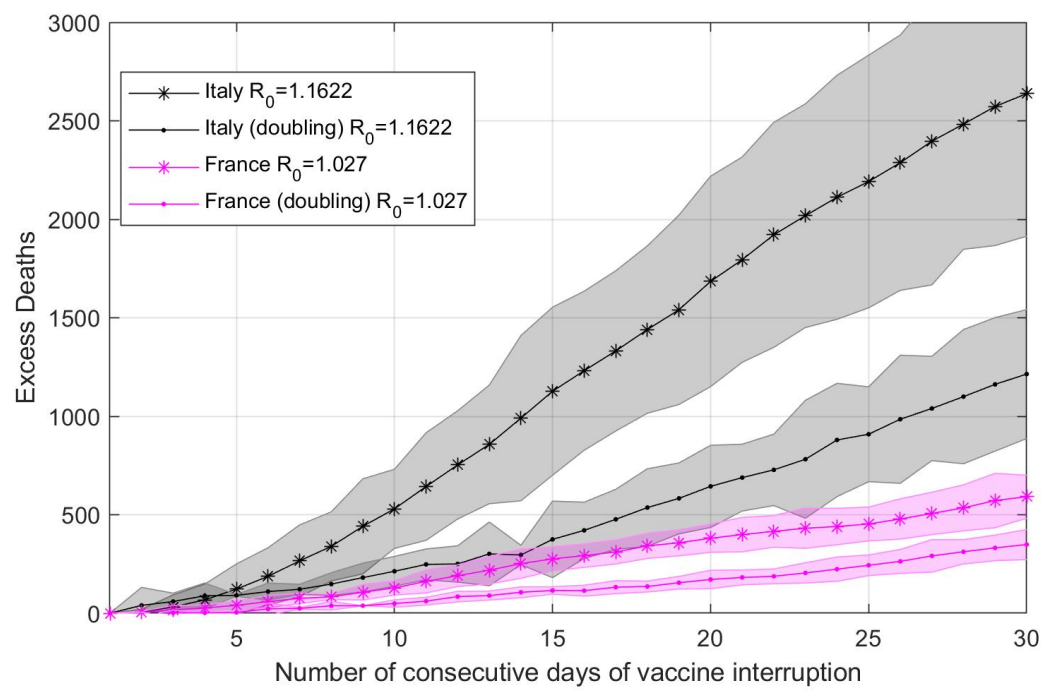

FIG. 3. Average over $N_{r}=1000$ realizations of the stochastic SEIR model showing the excess deaths $m \times I(t)$ as a function of the number of the days of interruption of AstraZeneca vaccinations for Italy $R_{0}=1.16$ (black and France $R_{0}=1.02$ (magenta). Simulations are smoothed with a moving average filter with window size 10 days. Stars indicate simulations where vaccination are resumed at the same rate, dots simulations where the vaccination rate is doubled for a number of days equivalent to those of interruption. Error bars are computed as the mean relative error.

number of 5 millions vaccinated people with AstraZeneca in Europe[33]. By suspect cases we mean people who have developed DVT in the few days following the vaccination. This leads us to an estimate of a frequency of 6 cases per million of vaccines. Let us call this rate $r_{A Z}^{D V T}$. Let us also consider that, in the case of France, the incidence of DVT has been estimated to 1800 people per 1 million inhabitants per year ([34]), with a lethality rate after three months of $5 \%$ [35], raising to $30 \%$ when a period of 5 years is considered [7]. This leads to estimating a total of the order of 10000 deaths per year as a result of DVT. Even assuming that all DVT cases following the inoculation of the AstraZeneca vaccine would have not manifested themselves in absence of the injection, we have that $N$ vaccinations would lead to an extra $N \times r_{A Z}^{D V T}$ DVT cases. Let us assume that all of these cases result into death[36]. We then have that $10^{5}$ daily vaccinations would result into a maximum of 0.6 daily deaths. In 500 days, which is the time needed to cover the entirety of the French population, this leads to an upper bound of 300 deaths. Considering a death rate of 30\%, the number scales down to approximately 100, while considering a death rate of $5 \%$ the 
number scales down to approximately 15. Similar figures apply for Italy.

\section{CONCLUSION}

Decision-making in presence of strong uncertainties associated with health and environmental risks is an extremely complex process, resulting from the interplay between science, politics, stakeholders, activists, lobbies, media, and society at large [37-39]. In this letter, we have aimed at contributing to the debate on different strategies for combating, in conditions of great uncertainties in terms of health and social response, pandemic like the current one caused by the SARS-CoV-2 virus. We have focused on the case of the AstraZeneca COVID-19 vaccine and on the locales of Italy and France, for the period starting on March 15th 2021. The goal is providing a semi-quantitative comparison, based on Fermi estimates informed by a simple yet robust stochastic model, between the excess deaths due to tempo-

ral restriction in the deployment of a still experimental vaccine and the excess deaths due to its possible side effects. Given the many uncertainties on the (possible) side effects of the vaccine, we have resorted to making worst case scenario calculations in order to provide a robust upper bound to the related excess deaths. Our results are preliminary and should be supplemented by more detailed modelling and data collection exercises. Indeed: i) we assume a single vaccine with the nominal AstraZeneca efficacy, neglecting the other available vaccines, ii) we consider a fixed vaccination rate, iii) for AstraZeneca DVT side effects we consider French data and rescale them for the Italian populations, iv) we focused our analysis on DVT side effects, but other pathologies could be considered with the same approach. Yet, these results clearly suggest - see a useful summary in Table II - that the benefits of deploying the vaccine greatly outweigh the associated risks, and that the relative benefits are wider in situations where the reproduction number is larger, and they increase with the temporal duration of the vaccine ban. We have also analysed the case of resuming the vaccinations at a double rate $\left(2 \cdot 10^{5}\right.$ vaccinations/day) for an amount of days equal to vaccine interruption period (Fig. 3 and Fig. S3). This analysis has pointed out that excess deaths are still of the same order of magnitude as those observed by resuming vaccinations with $10^{5}$ vaccinations/day injection rate but scale down by a factor 2 . This is an evident outcome of the nonlinear effects of epidemiological dynamics: those who have not been vaccinated can contaminate other individuals before vaccination resume, as a result of a cascade 
mechanism also observed in turbulent flows: there, energy injected in large scales vortex is transferred to small scales via nonlinear interactions between scales [40]. Here, in analogy, a few non-vaccinated individuals can produce a large number of infected individuals. The process can only stop if a huge number of daily vaccinations (much larger than a factor 2 ) is performed. Nevertheless, this still requires a characteristic recovery timescale $T$ that is larger than the typical immunization scale $\eta$ (e.g., a few months for AstraZeneca [28]). Finally, even if several countries have resumed, or are going to resume, AstraZeneca vaccinations, the effect of the interruption is hard to counterbalance and require vaccination efforts difficult to set-up in due times. Furthermore, at least for large countries where AstraZeneca vaccination could resume, the confidence of the population in the vaccines is reduced by a non negligible percentage [41]. In this sense, our estimates are likely to be conservative and might possibly underestimate the excess deaths deriving from the disbelief in the vaccination policies observed in the largest European countries. The analysis presented here has been performed with a parsimonious but well-posed and tested model and we hope that the results we obtain might be the starting point for more detailed, more advanced, and more mature investigations with sophisticated models and data collection exercises.

\section{ACKNOWLEDGEMENTS}

This work has been greatly supported by the London Mathematical Laboratory and we acknowledge the logistic support of SCuP. VLu acknowledges the support received from the EPSRC project EP/T018178/1 and from the EU Horizon 2020 project TiPES (Grant no. 820970). This is TiPES' contribution \#97. We thank A Veber, A Mazaud, FM Breon, the Modcov19 CNRS community and two anonymous reviewers for useful comments and suggestions.

\section{DATA AVAILABILITY}

Raw data that support the findings of this study are openly available in Johns Hopkins University Center for Systems Science at https ://systems . jhu .edu/research/public-health/ncov/. Derived data supporting the findings of this study are available from the corresponding au- 


\begin{tabular}{|c|c|c|}
\hline Excess Deaths & Italy & France \\
\hline Stop AZ for $t=500$ days & $9000 \pm 3000$ & $1200 \pm 400$ \\
\hline Stop AZ for $t=14$ days & $1700 \pm 500$ & $430 \pm 70$ \\
\hline Stop AZ for $t=7$ days & $790 \pm 90$ & $160 \pm 30$ \\
\hline Stop AZ for $t=3$ days & $260 \pm 50$ & $130 \pm 20$ \\
\hline Worst case DVT deaths due to AZ & $\approx 280$ & $\approx 300$ \\
\hline High fatality DVT deaths due to AZ & $\approx 90$ & $\approx 100$ \\
\hline Standard fatality DVT deaths due to AZ & $\approx 13$ & $\approx 15$ \\
\hline
\end{tabular}

TABLE II. The first 4 lines of the table indicate the excess deaths due to the interruption of AstraZeneca compared to a reference scenario where the vaccine injections are never interrupted. The SEIR model is integrated for 500 days with $R_{0}=1.16$ for Italy and $R_{0}=1.02$ for France. The last 3 rows of the table show the deaths from deep vein thrombosis (DVT) that could be due to the vaccine in three different scenarios: the worst case (100\% mortality rate), a high mortality scenario (death rate of $30 \%$ ) and a standard mortality scenario (5\% mortality rate) assuming a period of 500 days.

thor upon reasonable request.

[1] J. Wu, W. Cai, D. Watkins, and J. Glanz, The New York Times (2020).

[2] R. M. Anderson, H. Heesterbeek, D. Klinkenberg, and T. D. Hollingsworth, The Lancet 395, $931(2020)$.

[3] M. Chinazzi, J. T. Davis, M. Ajelli, C. Gioannini, M. Litvinova, S. Merler, A. Pastore y Piontti, K. Mu, L. Rossi, K. Sun, C. Viboud, X. Xiong, H. Yu, M. E. Halloran, I. M. Longini, and A. Vespignani, Science 368, 395 (2020), https://science.sciencemag.org/content/368/6489/395.full.pdf.

[4] H.-Y. Yuan, G. Han, H. Yuan, S. Pfeiffer, A. Mao, L. Wu, and D. Pfeiffer, medRxiv (2020), 10.1101/2020.05.03.20089482, https://www.medrxiv.org/content/early/2020/05/06/2020.05.03.20089482.full.pdf. 
[5] D. Sridhar and D. Gurdasani, Science $\mathbf{3 7 1 ,} 230 \quad$ (2021), https://science.sciencemag.org/content/371/6526/230.full.pdf.

[6] N. Fernandes, Available at SSRN 3557504 (2020).

[7] M. Cushman, Seminars in hematology 44, 62 (2007).

[8] Bloomberg, March 2021: https://tinyurl.com/2zk29abr.

[9] ANSA, March 2021: https://tinyurl.com/4ywxt5kp.

[10] K. Steele, Law, Probability and Risk 5, 19 (2006), https://academic.oup.com/lpr/articlepdf/5/1/19/6207484/mgl010.pdf.

[11] D. Faranda and T. Alberti, Chaos 30, 111101 (2020), arXiv:2006.05081 [q-bio.PE].

[12] T. Alberti and D. Faranda, Communications in Nonlinear Science and Numerical Simulation 90, $105372(2020)$.

[13] I. M. Held, Bulletin of the American Meteorological Society 86, 1609 (2005).

[14] M. Pascual, M. Roy, and K. Laneri, Theoretical Ecology 4, 211 (2011).

[15] U. Gähde, S. Hartmann, and J. Wolf, Models, Simulations, and the Reduction of Complexity, Abhandlungen der Akademie der Wissenschaften in Hamburg (De Gruyter, 2013).

[16] P. Almaraz, Frontiers in Ecology and Evolution 2, 54 (2014).

[17] M. Ghil and V. Lucarini, Rev. Mod. Phys. 92, 035002 (2020).

[18] S. Mahajan, The Art of Insight in Science and Engineering: Mastering Complexity (The MIT Press, Cambridge, USA, 2014).

[19] T. G. Shepherd, Current Climate Change Reports 2, 28 (2016).

[20] P. M. Anderson and C. A. Sherman, Journal of Applied Business \& Economics 10 (2010).

[21] D. Faranda, I. P. Castillo, O. Hulme, A. Jezequel, J. S. W. Lamb, Y. Sato, and E. L. Thompson, Chaos: An Interdisciplinary Journal of Nonlinear Science 30, 051107 (2020), https://doi.org/10.1063/5.0008834.

[22] F. Brauer, in Mathematical epidemiology (Springer, 2008) pp. 19-79.

[23] C. Sun and Y.-H. Hsieh, Applied Mathematical Modelling 34, 2685 (2010).

[24] G. E. Uhlenbeck and L. S. Ornstein, Phys. Rev. 36, 823 (1930).

[25] Paul L. Delamater et al., "Complexity of the basic reproduction number (r0) - volume 25, number 1-january 2019 - emerging infectious diseases journal - cdc," (2019).

[26] L. Salvioli, "Il vaccino anti covid in italia in tempo reale: Il sole 24 ore," (2021). 
[27] S. A. Lauer, K. H. Grantz, Q. Bi, F. K. Jones, Q. Zheng, H. R. Meredith, A. S. Azman, N. G. Reich, and J. Lessler, Annals of Internal Medicine (2020).

[28] Merryn Voysey et al., The Lancet, The Lancet 397, 99 (2021).

[29] D. Fanelli and F. Piazza, Chaos Solitons and Fractals 134, 109761 (2020), arXiv:2003.06031 [q-bio.PE].

[30] G. Pullano, L. Di Domenico, C. E. Sabbatini, E. Valdano, C. Turbelin, M. Debin, C. Guerrisi, C. Kengne-Kuetche, C. Souty, T. Hanslik, et al., Nature 590, 134 (2021).

[31] H. Risken, The Fokker-Planck equation (Springer, Berlin, 1996).

[32] The Guardian, March 2021: https://tinyurl.com/vvwhcydz.

[33] Here, we use European data accessible via the website of the European Medicines Agency at https://tinyurl.com/ht8y98kr to average out the large spread of national data.

[34] S. Bouée, C. Emery, A. Samson, J. Gourmelen, C. Bailly, and F.-E. Cotté, Thrombosis Journal 14, 4 (2016).

[35] J. A. Heit, Nature Reviews Cardiology 12, 464 (2015).

[36] Current data suggest that this is manifestly a gross worst case approximation.

[37] Anonymous, Eos, Transactions American Geophysical Union 91, 248 (2010), https://agupubs.onlinelibrary.wiley.com/doi/pdf/10.1029/2010EO280005.

[38] A. Benessia and B. De Marchi, Futures 91, 35 (2017), post-Normal science in practice.

[39] J. Reis and P. S. Spencer, Environmental Health and Preventive Medicine 24, 57 (2019).

[40] A. Kolmogorov, Akademiia Nauk SSSR Doklady 30, 301 (1941).

[41] The Economist, March 2021: https://tinyurl.com/83cbr4d3. 


\title{
Supplemental Material for: Interrupting vaccination policies can greatly spread SARS-CoV-2 and enhance mortality from COVID-19 disease: the AstraZeneca case for France and Italy
}

\author{
Davide Faranda* \\ Laboratoire des Sciences du Climat et de l'Environnement, \\ CEA Saclay l'Orme des Merisiers, UMR 8212 CEA-CNRS-UVSQ, \\ Université Paris-Saclay \& IPSL, 91191, Gif-sur-Yvette, France \\ London Mathematical Laboratory, 8 Margravine Gardens, London, W6 8RH, UK and \\ LMD/IPSL, Ecole Normale Superieure, \\ PSL research University, 75005, Paris, France \\ Tommaso Alberti \\ National Institute for Astrophysics-Institute for \\ Space Astrophysics and Planetology (INAF-IAPS), \\ via del Fosso del Cavaliere 100, 00133, Rome, Italy \\ Maxence Arutkin \\ UMR CNRS 7083 Gulliver, ESPCI Paris, \\ 10 rue Vauquelin, 75005 Paris, France \\ Valerio Lembo \\ Institute of Atmospheric Sciences and Climate (ISAC-CNR), Bologna, Italy \\ Valerio Lucarini \\ Department of Mathematics and Statistics, \\ University of Reading, Reading, UK and \\ Centre for the Mathematics of Planet Earth, \\ University of Reading, Reading, UK
}

(Dated: March 30, 2021) 


\begin{abstract}
The supplemental Material for "Interrupting vaccination policies can greatly spread SARS-CoV2 and enhance mortality from COVID-19 disease: the AstraZeneca case for France and Italy" contains: i) the numerical code used in this study, ii) three supplementary figures.
\end{abstract}

\footnotetext{
*davide.faranda@cea.fr
} 


\section{NUMERICAL CODE}

\%Numerical SEIR code for "Interrupting vaccination policies can greatly spread \%SARS-CoV-2 and enhance mortality from COVID-19 disease: the AstraZeneca case \%for France and Italy" by Faranda et al.

\%This code integrates the SEIR Model with vaccination policies interrupted for 7 days. $\%$ The data are referred to the Italian population

Country=' Italy';

\%Population;

$\mathrm{N}=60000000$;

$\%$ Initial conditions

$\mathrm{S}(1)=\mathrm{N}-9000000-2000000 ; \%$ Susceptibles, including those

$\%$ who had the virus in the first and second wave (9 millions)

$\%$ and those who received two doses vaccines

$E(1)=20000 ; \%$ Exposed

$I(1)=20000 ; \%$ Infected

$R(1)=11000000 ; \%$ Recovered

$\%$ Parameters

alpha0 $=100000 . / \mathrm{N} ; \%$ Vaccination rate

sigma0 $=0.59 ; \%$ Vaccine Efficacy

epsilon $0=0.27$; Incubation Rate

beta $0=0.37$; Recovery Rate

lambda0 $=0.45 \%$ Infection Rate

mort0 $=0.015 ; \%$ Mortality

$\mathrm{R} 0=1 \mathrm{ambda0} . /$ beta0; \%Basic Reproduction number

\% Dynamical steps

t_susp $=7$; \%example where the vaccination is suspended for one week Tint $=500$; 
for $t=1:$ Tint

$\% \mathrm{~F}$

if $t<t_{\text {s susp }}$

alpha $=0$;

sigma $=0$;

epsilon=epsilon 0 +0.2*epsilon $0 *$ randn;

beta $=$ beta $0+0.2 *$ beta $0 *$ randn;

lambda $=1$ ambda $0+0.2 * l$ ambda $0 *$ randn;

else

alpha $=a l$ pha $0+0.25 * a l$ pha0 0 randn;

sigma $=$ sigma $0+0.1 *$ sigma $0 *$ randn;

epsilon=epsilon 0 +0.2*epsilon $0 *$ randn;

beta $=$ beta $0+0.2 *$ beta $0 *$ randn;

lambda $=1 \mathrm{ambda} 0+0.2 * 1 \mathrm{ambda} 0 * \mathrm{randn}$;

end

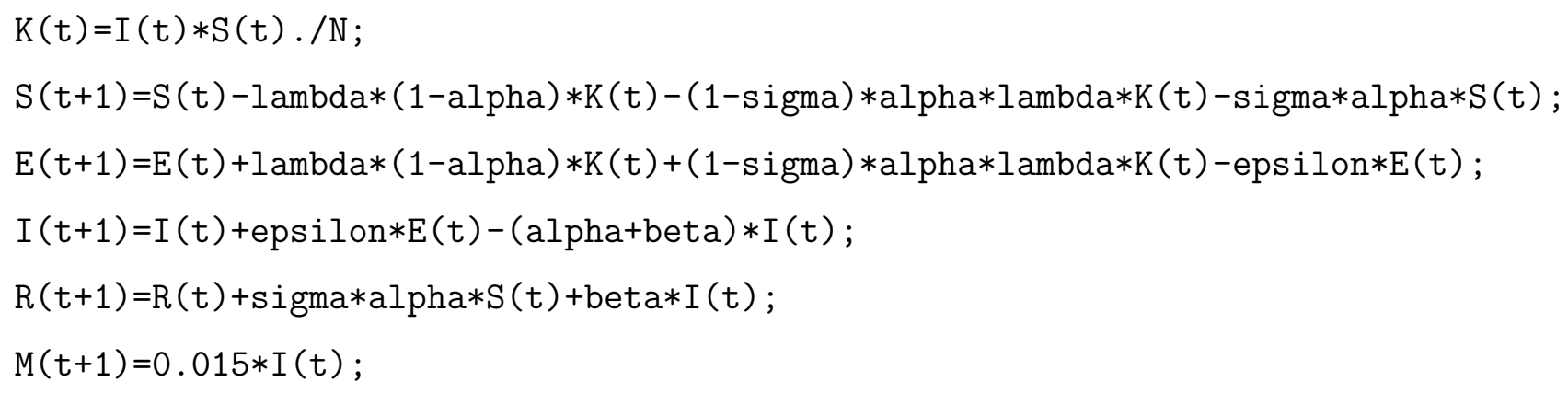

end

\section{SUPPLEMENTARY FIGURES}



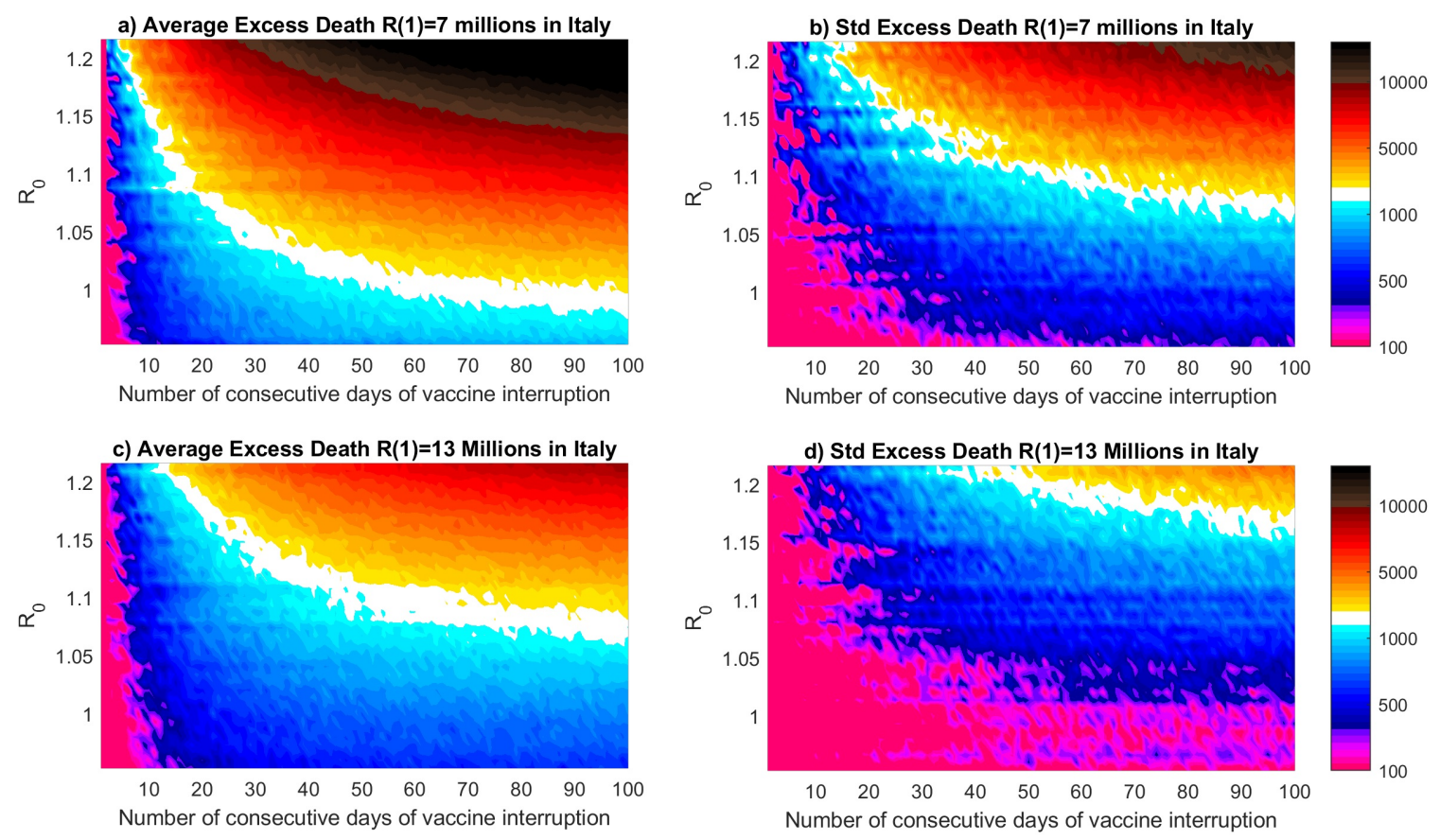

FIG. 1. (a,c) Average and (b,d) standard deviation over $N_{r}=1000$ realizations of the SEIR model showing the excess deaths $m \times I(t)$ as a function of the number of the days of interruption of AstraZeneca vaccinations (x-axis) and $R_{0}$ (y-axis) for Italy with $R(1)=7$ millions (a,b) and $R(1)=13$ millions $(\mathrm{c}, \mathrm{d})$. The excess deaths are computed with respect to a base scenario where vaccine injections are never interrupted. Arrows indicate the values of $R_{0}$ chosen for Figs. 2 and 3. The model is integrated for 500 days. $x$-axis starts at $N=1$ 


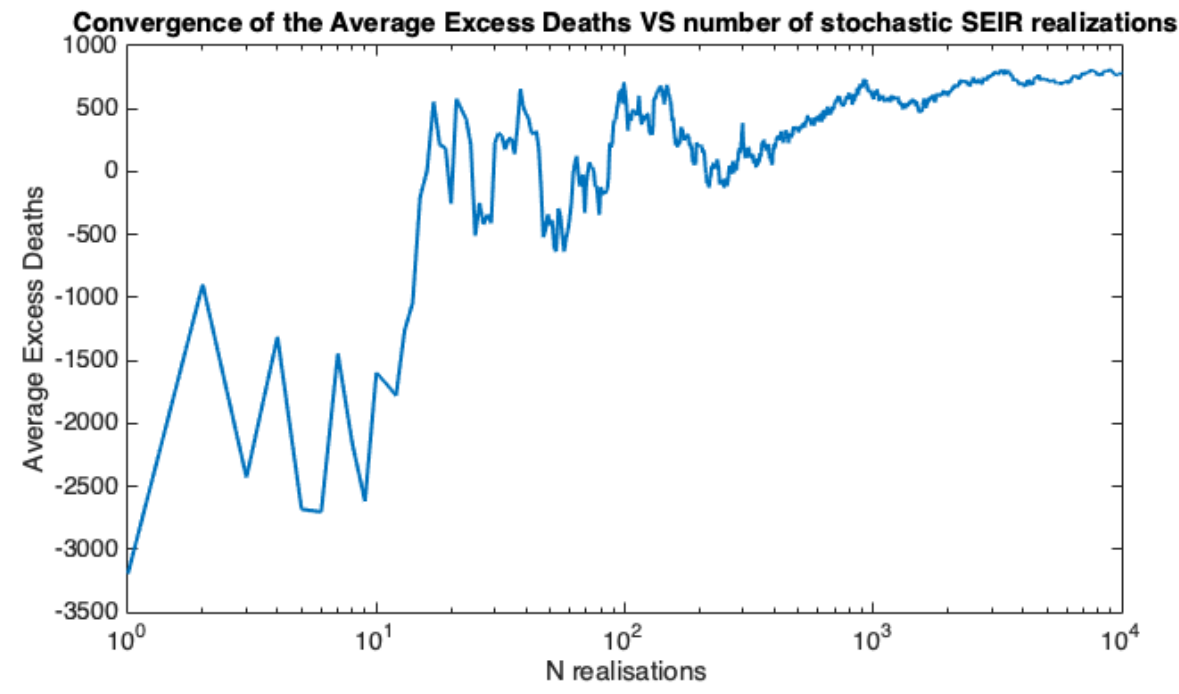

FIG. 2. Convergence in number of realisation $N_{r}$ (x-axis) of the average excess deaths (y-axis) in Italy after a 5 days interruption of AstraZeneca vaccinations. The excess deaths are computed with respect to a base scenario where vaccine injections are never interrupted. The average excess deaths achieve a good convergence at $10^{3}$ realizations. 

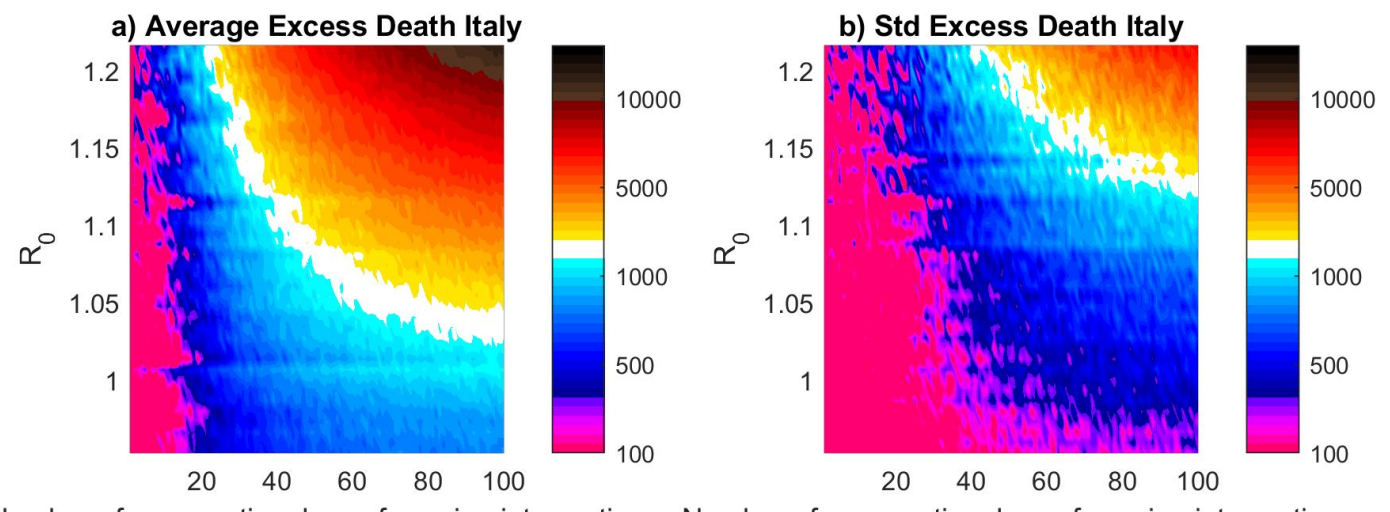

Number of consecutive days of vaccine interruption Number of consecutive days of vaccine interruption
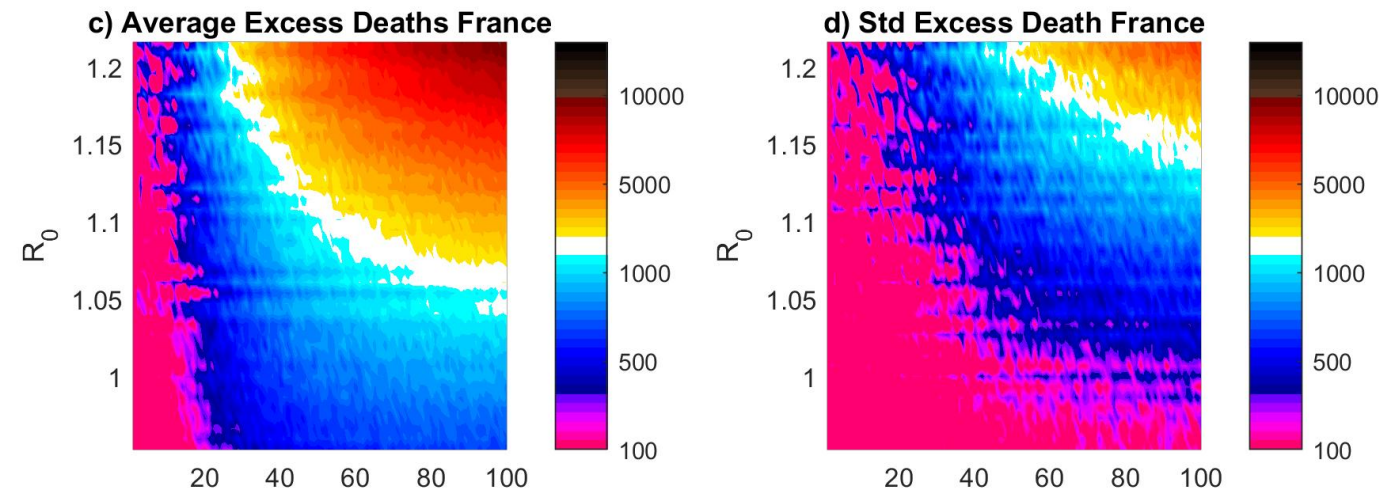

Number of consecutive days of vaccine interruption Number of consecutive days of vaccine interruption

FIG. 3. (a,c) Average and (b,d) standard deviation over $N_{r}=1000$ realizations of the stochastic SEIR model showing the excess deaths $m \times I(t)$ as a function of the number of the days of interruption of AstraZeneca vaccinations (x-axis) and $R_{0}$ (y-axis) for Italy (a,b) and France (c,d). The excess deaths are computed with respect to a base scenario where vaccine injections are never interrupted. With respect to Figure 2 in the main text, here the vaccination rate is doubled for a number of days equivalent to those of interruption. Note that $x$-axis starts at $N=1$. Each realization of the SEIR model is integrated for 500 days. 